\title{
Non Equilibrium Thermodynamic Studies Through Activation Parameters In Transport Phenomenon Across Ion Exchange Membranes.
}

\author{
Dr. Kalpana Virendra Singh \\ P.G. Department of Chemistry and Pharmaceutical Chemistry Govt. Madhav Science P.G.College Ujjain (M.P.)
}

\begin{abstract}
Transport phenomenon across membranes are very important as they work as guiding principles for drug permeation studies across biological membranes. Classical thermodynamics is confined to the systems of equilibrium i.e. to reversible processes only and cannot be applied to the irreversible systems where variables defining the systems are changing with time and situation. The utility of irreversible thermodynamics lies in the evaluation of entropy production by use of the equation for conservation of mass energy and Gibb 'sequation.. In the present investigation membrane is prepared by mechanical compression of cation exchange resin Indion 236 with adhesive araldite and has been characterized in terms of membrane constant A/l.Filteration Coefficient $L_{P}$ for different concentrations of aqueous solutions of glucose and sucrose at different temperatures have been determined. The present study tries to calculate values of Gibb's free energy, entropy and enthalpy for transport across ion exchange membrane using values of filteration coefficient $L_{P}$ equivalent pore radius, concentration of solutes and temperature of study.
\end{abstract}

Keywords: Transport, non-equilibrium thermodynamics, Gibb's free energy, entropy, enthalpy

\section{Introduction}

Transport phenomenon across natural and artificial membranes arise because of the urge of a system to move from non equilibrium to equilibrium state. Transport phenomenon across membranes may be active transport ,passive transport, facilitated transport or group translation depending upon the nature of membrane , size and shape of the permeant molecules and nature of interface charged or otherwise [1-6].Transport phenomenon across membranes can be best interpreted with the help of the theory of non-equilibrium thermodynamics[15]

Ion Exchange membranes are highly charged artificial membranes withfixed ionic groups, mobile ions in the close vicinity bearing the same charge as that the fixed ions are known as co-ions and the mobile ions bearing the opposite charges are known as counter ions. Structurally ion exchange membranes have characteristic pore size capillaries or channels, chemically the matrix consists of an irregular, macromolecular, three dimensional net work with charged ionic groups in its component polymer molecules. Electrical double layers are formed at the interface of ion Exchange membranes which involve the reversible interchange of ions between solid and liquid phase. Ion Exchange membranes have been used as simple models for the study of biological processes across biomembranes.

Fick's law is the basic principle which describes free diffusion in one dimension in a solution and can be stated as

$J_{0}=-\mathrm{D}\left(\delta_{c} / \delta_{x}\right)$.

Where $J_{0}$ is solute flux in moles per unit, $\mathrm{c}$ is concentration, $\mathrm{x}$ is distance and $\mathrm{D}$ is the diffusion coefficient in free solution. There exist friction of particles within the membrane pores. Pepenheimer et.al. [7] used following equation derived by Ladenburg to describe the friction of particles within the membrane pores. $g^{\prime} / g^{0^{\prime}}=1+2.4 \alpha$

$g^{\prime}$ is friction exerted on solute molecule as a consequence of interaction with in the pore. $\alpha$ is ratio of the radius of solute to pore.

Filteration Coefficient $L_{P}$ is a measure of a membrane's permeability, it denotes the volume of fluid filtered per unit time per unit area of membrane per unit pressure difference. Both hydraulic and osmotic pressures are taken into account. The volume flow $J_{V}$ as a function of the applied pressure difference $\Delta \mathrm{P}$ and osmotic pressure difference $\Delta \pi$ across the membrane is given by using Starling equation

$J_{V}=L_{P} \Delta \mathrm{P}-L_{P} \Delta \pi \sigma \ldots \ldots \ldots \ldots \ldots \ldots \ldots \ldots .$.

Where $J_{V}$ is volume flow

$L_{P}$ isFilteration Coefficient 
$\Delta \mathrm{P}$ is applied pressure difference

$\Delta \pi$ is osmotic pressure difference

It is convenient to determine the filtration coefficient $L_{P}$ at $\Delta \pi=0$, which can be achieved by taking equal concentrations of the solute on both sides of the membrane, so that (1) can be written as

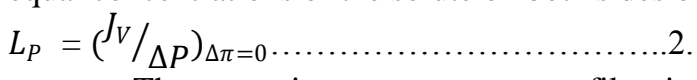

The experiments to measure filtration coefficient in biological systems were performed by Pappenheimer, Renkin, and Borrero $[8,9,10$,$] to determine changes in tissue fluid content$

Rejection or coupling coefficient $\sigma$

Rejection or coupling coefficient $\sigma$, is the measure of the membrane selectivity and according to Staverman [16] when $\sigma=1$ all the solute is rejected from the membrane, while $\sigma<1$ means a part of the solute passes through the membrane. According to the pore model of the transport of solutions through the membranes, rejection coefficient is also known as the coupling coefficient.

Rejection/coupling coefficient can be estimated from equation 1 .

$J_{V}=L_{P}(\Delta \mathrm{P}-\Delta \pi \sigma) \ldots \ldots \ldots \ldots \ldots \ldots \ldots \ldots \ldots \ldots .$.

Since $\Delta \pi=\mathrm{RT} \Delta C$

$J_{V}=L_{P}(\Delta \mathrm{P}-\sigma \mathrm{RT} \Delta C)$

As per equation 4 the plot of $J_{V}$ versus $\Delta \mathrm{P}$ should be a straight line.

If the hydrodynamic pressure is kept constant i.e. $\Delta P=0$, then equation 4 can be written as

$\left(J_{V}\right)_{\Delta P=0}=-\sigma L_{P}$ RT $\Delta C$

$\sigma=-\left[\frac{J_{V}}{L_{P} \mathrm{RT} \Delta C}\right]$

Negative sign is indicative that the hydrostatic and osmotic forces act in the opposite direction.

Irreversible thermodynamics explains that the hydrodynamic flow of solution through a porous medium can be either viscous flow, diffusional flow or combination of both. These flows vary exponentially with temperature[17] and are characterized in terms of activation energy and hence free energy[18]

$\left(J_{V}\right)_{\Delta \emptyset=0}=L_{P} \Delta P$ ...5

For above equation to be compatible with Poissuille's law

$L_{P}=\pi \sum_{i=1}^{i=n} \frac{r_{i}^{4}}{8 \dot{\eta} l}$

Where $\mathrm{r}$ is the radius of the ith capillary, $\mathrm{n}$ number of capillaries and $\dot{\eta}$ is the viscosity. Variation of viscosity with temperature can be expressed as an activation process.

$\grave{\eta}=\mathrm{A} e^{E_{n} / R T}$ . .7 .

$E_{n}$ being activation energy

Entropy of activation can be calculated using Eyring rate equation

$\dot{\eta}=\frac{N h}{V} e^{-\Delta S * / R} e^{\Delta H * / R T}$. ....8.

$\Delta S *=\frac{\Delta H}{T}+\mathrm{R} \log \left(\frac{N h}{\eta V}\right) \ldots \ldots \ldots \ldots \ldots .$.

$\Delta \mathrm{H}^{*}$ and $\Delta \mathrm{S}^{*}$ are related to $\Delta \mathrm{G}^{*}$ as

$\Delta \mathrm{G}^{*}=\Delta \mathrm{H}^{*}-\mathrm{T} \Delta \mathrm{S}^{*}$ . .10.

Negative value of $\Delta \mathrm{G}^{*}$ points towards spontaneity, where as positive value shows that the flow is not facilitated favoured across the membrane, the flow process is non-spontaneous

\section{1 materials}

\section{Experimental}

\subsection{1chemical reagents}

Sucrose and Glucose of analytical grade and used as such after drying over $\mathrm{P}_{2} \mathrm{O}_{5}$ in a vacuum dessicator.

\subsection{2 water}

Water required for the preparation of solutions and for the calibration of Viscometer and Pycnometer was prepared by distilling twice in an all glass double distillation unit supplied by Systronics India Ltd. Specific conductance of water thus prepared was of the order of $10^{-6} \mathrm{ohm}^{-1} \mathrm{~cm}^{-1}$. Water was stored in Borosilicate glass bottles. 


\subsection{3 cation exchange resin}

Indion 236 from sd fine Chemicals India was used for the preparation of membrane.

\subsection{Preperation of membrane}

The cation exchange resin (Indion 236)was swollen in conductivity water and casted in the form of plug as described below

$9 \mathrm{gm}$. of ion-exchange resin along with small amount of (5-7\%)of an adhesive (araldite )was placed in a Pyrex glass assembly having constriction in the middle and compressed mechanically at the site of constriction with the help of mechanical device consisting of wooden rods having diameter slightly less than that of glass tube. The screws of the device were tightened and assembly was left as such for 24 hours for the complete setting of the plug. The thickness and diameter of the plug thus prepared were $2.29 \mathrm{cms}$ and $1.398 \mathrm{cms}$ respectively. The maximum variation in the permeability of the ion -exchange membrane, thus prepared for a period of one week was only of the order of $5 \%$.

In order to know about the directional character of the membrane for the permeation of water the hydraulic permeability was measured in both the directions at $35^{\circ} \mathrm{C}$ and the values were found to be same in both the directions, thereby indicating the isotropic character of the membrane.

\section{3 apparatus}

The apparatus consists of a pyrex glass tube of $24 \mathrm{cms}$. In length having a slight constriction in the middle with an internal diameter $1.398 \mathrm{~cm}$, where the plug of cation exchange resin is set up.This tube has two standard female joints B-24 at the ends. To the standard B-24 male joints are fixed the coiled platinum electrodes $\mathrm{F}$ and $\mathrm{G}$. The ends of the electrodes are fused in glass tubes of diameter $5 \mathrm{~mm}$. so that the electrode ends are insulated from the permeant. The lengths of these glass tubes are adjusted in such a way that when standard joints are kept in position, the electrodes touch the cross-sectional surface of the membrane. The main tube has two side tubes $\mathrm{H}$ and $\mathrm{K}$, bearing B-14 female standard joints D and E. Through the joint E a capillary tube $\mathrm{J}$, of known diameter, bent at $90^{\circ}$ of length $25 \mathrm{~cm}$. is connected to the side tube $\mathrm{K}$. A graduated tube I of about $30 \mathrm{cms}$ in length and $1.0 \mathrm{~cm}$. in diameter is connected to the side tube $\mathrm{H}$, through another standard joint D.The design of apparatus and experimental set up is shown in "Fig"1

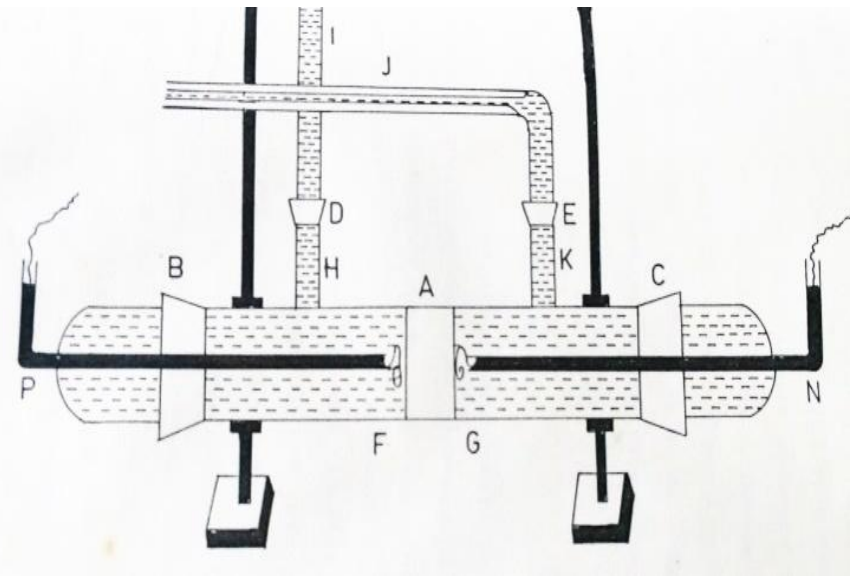

fig.1. schematic set up of the apparatus.

\section{4 working procedure}

The experimental cell was filled with water and left overnight for equilibration of the plug. The cell was then thoroughly washed with fresh distilled water under pressure gradient to ensure thorough cleanliness. The cell was then filled, by adding the solution under investigation on both sides of the membrane and left overnight. Next day the solution was thrown. The apparatus was then filled by adding the solution under investigation on one side of the membrane and then forcing it to the other side under the pressure gradient by vacuum pump. This ensures the complete filling of the capillaries of the membrane. The whole apparatus was then kept in air thermostat maintained at the desired temperature with in ${ }_{-}^{+} 0.05^{\circ} \mathrm{C}$. For the measurement of hydrodynamic permeability, desired pressure difference was applied across one side of the cell with the help of a pressure head.The system was kept in the thermostat for about two hours to allow the experimental solution to attain the temperature of the thermostat. At desired pressure difference, the rate of flow of liquid was measured by noting the time taken by the solution to move a certain distance i.e one $\mathrm{cm}$. through horizontal capillary. Time of flow was recorded by using a stopwatch of least count $0.1 \mathrm{~s}$. The flow was recorded at different pressures and temperatures. 
Conductance of the system and specific conductance was noted with the help of Digital conductivity meter. The conductivity cell consisted of two platinum electrodes fused with glass.Density of the solution is noted with pre calibrated Pycnometer and viscosity was measured with a suspended lebel type viscometer.

\subsection{Sources of Error}

The main source of error and factor responsible for affecting reproducibility of results is the incomplete wetting of the membrane, as rate of flow of liquid depends upon the actual number of capillaries transmitting the liquid. This was ensured by introducing the solution after evacuation of the apparatus and by preparing the solution from degassed water

\section{Results And Discussion}

Indion 236 ion exchange membrane is characterized before putting into the practical use. Effective cross-sectional area, equivalent pore radius and the electrical character of the membrane is determined.

\subsection{Membrane Characterization}

The thickness of the membrane is measured with the help of cathetometer of $0.001 \mathrm{~cm}$.measurement limits and found out to be equal to $2.29 \mathrm{~cm}$., Diameter of the membrane is noted with the help of travelling microscope of $0.001 \mathrm{~cm}$. sensitivity and found out to be equal to $1.398 \mathrm{~cm}$.

The rate of permeation through the membrane, under the influence of hydrostatic pressure depends upon the effective cross-sectional area, which is difficult to determine due to the complex geometry of the opening with in the membrane. However determination of the ratio A/l,so called membrane constant is possible , in terms of which the permeant behavior of any membrane can be expressed quantitatively.

For a membrane having ' $n$ ' pores of equivalent radius ' $r$ ', the effective cross-sectional area 'A', through which permeation occurs is $\mathrm{n} \pi r^{2}$. The electrical conductance $\mathrm{K}$ of the membrane equilibrated with a permeant having specific conductance $\mathrm{k}$ is given by

$\mathrm{K}=\mathrm{n} \pi r^{2} \cdot \mathrm{k} / \mathrm{l}=(\mathrm{A} / \mathrm{l}) \cdot \mathrm{k}$

So that the membrane constant is

$\mathrm{A} / \mathrm{l}=\mathrm{K} / \mathrm{k}$

Membrane constant is characteristic parameter of the membrane and is independent of the permeating liquid as long as the interaction between the permeant and the membrane matrix is not strong enough to alter pore radius. values of membrane constant are found out to be fairly constant for different solutions as listed in table 1. and are in accordance with the findings of singh et.al.[11]. Thus the membrane constant, A/l is a characteristic of membrane only and is independent of the nature of permeating liquid. The data is previously reported in the paper published by the author itself [12]

When net hydrodynamic flow $J_{V}$ is plotted against $\Delta P$ a straight line is obtained. Sample plot for sucrose at $308 \mathrm{~K}$ is given in Figure 2. The slope of the lines give filtration coefficient $L_{P}$ at different temperatures for various concentrations, intercept gives the value of net hydrodynamic flow at zero pressure difference. Experimental values are given in Table 1.

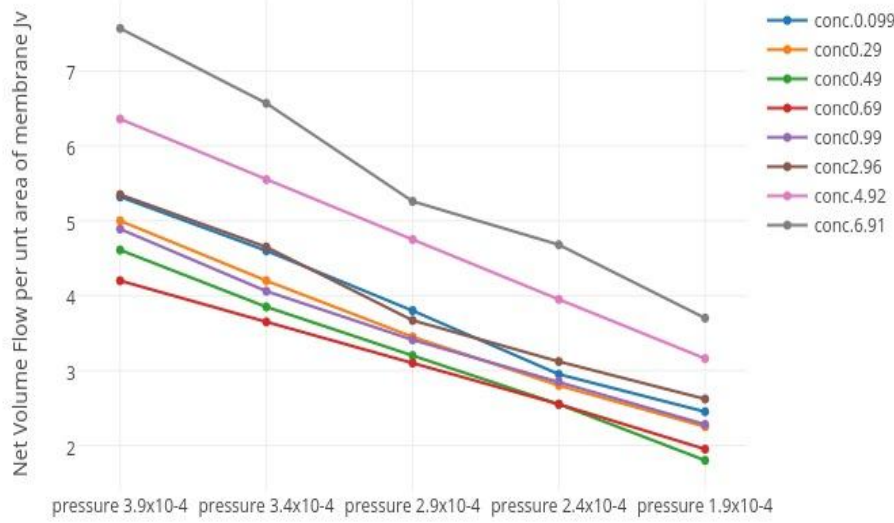

Figure $2 J_{V}$ versus $\Delta P$ for Sucrose at $308 \mathrm{~K}$, Sample plot 
Non Equilibrium Thermodynamic Studies Through Activation Parameters In Transport...

Table 1. Experimental values of net hydrodynamic flow $J_{V}$ at zero pressure difference and $L_{P}$ for various concentration at different temperatures across membrane

\begin{tabular}{|c|c|c|}
\hline $\begin{array}{l}\text { Concentration } \\
\Delta C \mathrm{X}^{2} 0^{2} \mathrm{moll}^{-1}\end{array}$ & $\begin{array}{l}J_{V} \times 10^{5} \\
\mathrm{~cm} \mathrm{sec}^{-1}\end{array}$ & $\begin{array}{l}L_{P} \mathrm{X}_{10}^{9} \\
\mathrm{~cm}^{3} \text { dyne }^{-1} \text { sec }^{-1} \\
\end{array}$ \\
\hline & Glucose in Water Temp. 308K & \\
\hline 0.099 & -0.55 & 1.58 \\
\hline 0.29 & -0.55 & 1.33 \\
\hline 0.49 & -0.60 & 1.30 \\
\hline 0.69 & -0.15 & 1.20 \\
\hline 0.99 & -0.15 & 1.27 \\
\hline 2.96 & -0.45 & 1.48 \\
\hline 4.92 & -0.10 & 1.64 \\
\hline \multirow[t]{2}{*}{6.91} & -0.05 & 1.89 \\
\hline & Glucose in Water Temp. 313K & \\
\hline 0.099 & -0.45 & 1.57 \\
\hline 0.29 & -0.45 & 1.67 \\
\hline 0.49 & -0.35 & 1.83 \\
\hline 0.69 & -0.40 & 2.06 \\
\hline 0.99 & -0.35 & 2.21 \\
\hline 2.96 & -1.25 & 0.09 \\
\hline 4.92 & -0.60 & 2.12 \\
\hline \multirow[t]{2}{*}{6.91} & -0.30 & 2.30 \\
\hline & Glucose in Water Temp. 318K & \\
\hline 0.099 & -2.40 & 0.92 \\
\hline 0.29 & -2.50 & 1.08 \\
\hline 0.49 & -2.50 & 1.21 \\
\hline 0.69 & -3.10 & 1.50 \\
\hline 0.99 & -6.20 & 1.62 \\
\hline 2.96 & -5.00 & 1.81 \\
\hline \multirow[t]{2}{*}{4.92} & -3.60 & 1.88 \\
\hline & Sucrose in Water Temp. 308K & \\
\hline 0.099 & -0.25 & 1.03 \\
\hline 0.29 & -0.20 & 1.14 \\
\hline 0.69 & -0.20 & 1.17 \\
\hline 0.99 & -0.15 & 1.25 \\
\hline 2.96 & -0.35 & 1.43 \\
\hline 4.92 & -0.15 & 1.50 \\
\hline \multirow[t]{2}{*}{6.91} & -0.45 & 1.80 \\
\hline & Sucrose in Water Temp. 318K & \\
\hline 0.099 & -0.40 & 1.63 \\
\hline 0.29 & -0.40 & 1.50 \\
\hline 0.49 & -0.45 & 1.43 \\
\hline 0.69 & -0.40 & 1.11 \\
\hline 0.99 & -0.10 & 0.75 \\
\hline 2.96 & -0.40 & 0.74 \\
\hline \multirow[t]{2}{*}{4.92} & -0.35 & 0.41 \\
\hline & Sucrose in Water Temp. 318K & \\
\hline 0.099 & -2.20 & 1.57 \\
\hline 0.29 & -1.10 & 1.08 \\
\hline 0.49 & -1.20 & 1.09 \\
\hline 0.69 & -1.50 & 1.00 \\
\hline 0.99 & -1.30 & 0.78 \\
\hline 2.96 & -1.30 & 0.65 \\
\hline 4.92 & -1.33 & 0.55 \\
\hline
\end{tabular}

The hydrodynamic flow through a membrane can be viscous or diffusional flow characterized by irreversible thermodynamics, varying exponentially with temperature. Dependence of these flows on temperature is characterized in terms of activation energy. In order to obtain the activation energy, hydrodynamic flow for different solutions of glucose and sucrose is studied at different temperatures. Values of $\log L_{P}$ for Sucrose and Glucose are given in table 2. Straight line is obtained when $\log L_{P}$ is plotted as a function of $\frac{1}{T}$. slope of the straight line gives the value of activation energy $E_{n}$. Values of Entropy and Gibb's free energy are calculated using equation 9. and 10.Values of activation energy, entropy and Gibb's free energy are given in table 3 . 
Non Equilibrium Thermodynamic Studies Through Activation Parameters In Transport...

Table II. Values of $\log L_{p}$ for different concentrations of Glucose and Sucrose in water at different temperatures

\begin{tabular}{|c|c|c|}
\hline $\begin{array}{l}\text { Concentration } \\
\mathrm{CX} 10^{2} \mathrm{~mol} l^{-1}\end{array}$ & $\log L_{p}$ Glucose & $\log L_{p}$ Sucrose \\
\hline & Temperature $308 \mathrm{~K}$ & \\
\hline 0.099 & -9.000 & -8.943 \\
\hline 0.29 & -9.041 & -9.000 \\
\hline 0.49 & -9.066 & \\
\hline 0.69 & -9.081 & -9.066 \\
\hline 0.99 & -8.889 & -9.036 \\
\hline 2.96 & -8.848 & -8.876 \\
\hline 4.92 & -8.833 & -8.777 \\
\hline \multirow[t]{2}{*}{6.91} & -8.742 & -8.770 \\
\hline & Temperature $313 \mathrm{~K}$ & \\
\hline 0.099 & -9.000 & -8.777 \\
\hline 0.29 & -8.963 & -8.851 \\
\hline 0.49 & -8.921 & -9.000 \\
\hline 0.69 & -8.857 & -9.027 \\
\hline 0.99 & -8.804 & -9.201 \\
\hline 2.96 & -8.796 & -9.259 \\
\hline 4.92 & -8.779 & -9.444 \\
\hline \multirow[t]{2}{*}{6.91} & -8.755 & -9.482 \\
\hline & Temperature $318 \mathrm{~K}$ & \\
\hline 0.099 & -8.769 & -8.712 \\
\hline 0.29 & -8.648 & -8.806 \\
\hline 0.49 & -8.542 & -8.808 \\
\hline 0.69 & -8.435 & -9.000 \\
\hline 0.99 & -8.684 & -9.066 \\
\hline 2.96 & -8.439 & -9.252 \\
\hline 4.92 & -8.273 & -9.301 \\
\hline 6.91 & -8.267 & -9.347 \\
\hline
\end{tabular}

\begin{tabular}{|l|l|}
\hline $\begin{array}{l}\text { Concentration } \\
\mathrm{CX} 10^{2} \mathrm{moll}^{-1}\end{array}$ & $\begin{array}{l}\text { Energy of activation } E_{n} \\
\mathrm{KJ}^{-1} \mathrm{~mol}^{-1}\end{array}$ \\
\hline \multicolumn{2}{|c|}{ Glucose in Water } \\
\hline 0.29 & 24.94 \\
\hline 0.49 & 36.74 \\
\hline 0.69 & 49.80 \\
\hline 2.96 & 31.17 \\
\hline 4.92 & 41.57 \\
\hline \multicolumn{2}{|c|}{ Sucrose in Water } \\
\hline 0.099 & 23.27 \\
\hline 0.49 & 14.10 \\
\hline 0.69 & 14.10 \\
\hline 0.99 & 22.86 \\
\hline 2.96 & 22.95 \\
\hline 6.91 & 21.12 \\
\hline
\end{tabular}

The positive values of $\Delta \mathrm{G}$ in all cases shows that the flow is not facilitated across the porous medium, i.e the flow process is non-spontaneous across the membrane, until an input force is applied across the membrane the flow of the permeantcan't take place.

\section{Conclusion}

In the present investigation cation exchange membrane has been prepared with Indion- 236.Transport studies viz. hydrodynamic permeabilities of aqueous solutions of glucose and sucrose in water across the membrane at various concentrations and different temperatures have been carried out. The membrane was characterized in terms of membrane constant $\mathrm{A} / \mathrm{l}$. As suggested by experimental data the membrane constant has been found to be fairly constant for glucose and sucrose solutions, indicating that the membrane constant is characteristic of the membrane only and is independent of the nature of the permeating liquid. It can be concluded from experimental data that hydrodynamic flow depends linearly on the pressure applied. However the values of reflection coefficient $L_{P}$ observed at different temperatures can be attributed to the different permeability of membrane with different solutes and concentrations at different temperature. The behavior can be attributed to different frictional forces which leads to change in the permeability of membrane reflected through values of equivalent pore radius and reflection coefficient. 


\section{References}

[1]. O.Brien, "Molecular anatomy of membranes" F.E.B.S. symposium, 20( 33) 1970

[2]. N. Lakshminarayanaiah, Chem. Rev., 2(203), 1970

[3]. N. Lakshminarayanaiah, "Transport through Membranes" ,Academic press,New York, (2), 1969.

[4]. H.T.Tien, "Bilayer Lipid Membranes, Marcel Dekker, Inc., New York,(4), 1974

[5]. K.Sollner, Ann. N.Y. Acad. Sci, 157 (177) 1953

[6]. F.Helfferich, "Ion - Exchange",McGraw Hill, New York,351, 1962

[7]. Peppenheimer J.R., E.M. Renkin and L.M. Borrero, “ Filteration ,diffusion and molecular sieving through peripheral capillary membranes",Am. J. physiol, 13(167) 1951

[8]. Pappenheimer JR. Passage of molecules through capillary wals. PhysiolRev , 33, 1953, 387-423.

[9]. Pappenheimer JR, Renkin EM, and Borrero LM. Filtration, diffusion and molecular sieving through peripheral capillary membranes; a contribution to the pore theory of capillary permeability. Am J Physiol 167,1951, $13-46$.

[10]. Pappenheimer JR and Soto-Rivera A. Effective osmotic pressure of the plasma proteins and other quantities associated with the capillary circulation in the hindlimbs of cats and dogs. Am J Physiol , 152,1948, . 471-91.

[11]. K.Singh, R.Kumar and U.N.Shrivastava, "Characterization of membranes"J.Indian Chem. Soc,58, 1980., 203.

[12]. KalpanaVirendra Singh Equivalent Pore Dimensions and Membrane Characterization parameters in Transport Phenomenon across Ion Exchange Membrane IOSR Journal of Applied Chemistry (IOSR-JAC),9(1) ,2016, 58-64.

[13]. Herbette LG, Rhodes DG, Mason RP. New approaches to drug design and delivery based on drug-membrane interactions. Drug Des Deliv ,7, 1991,75-118.

[14]. Seydel JK, Coats EA, Cordes HP, Wiese M. Drug membrane interaction and the importance for drug transport, distribution, accumulation, efficacy and resistance. Arch Pharm (Weinheim)327, 1994,601-10.

[15]. J.Th.G.Overbeek,J.Colloid Sci., 8,420,1935.

[16]. A.J.Staverman, "Theory of measurement of osmotic pressure,"Roc. Trav. Chim.,170, 344, 1951

[17]. S.Glasston, K.J.Laidler, H.Eyring, "Theory of rate processes”,McGraw Hill,(1941).

[18]. R.M.Barrer, "Diffusion in and through solids",Cambridge University Press(London),1951 